\title{
Acknowledgement to Reviewers of Laws in 2016
}

\author{
Laws Editorial Office \\ Published: 10 January 2017 \\ MDPI AG, St. Alban-Anlage 66, 4052 Basel, Switzerland; laws@mdpi.com
}

The editors of Laws would like to express their sincere gratitude to the following reviewers for assessing manuscripts in 2016.

We greatly appreciate the contribution of expert reviewers, which is crucial to the journal's editorial process. We aim to recognize reviewer contributions through several mechanisms, of which the annual publication of reviewer names is one. Reviewers receive a voucher entitling them to a discount on their next MDPI publication and can download a certificate of recognition directly from our submission system. Additionally, reviewers can sign up to the service Publons (https://publons.com) to receive recognition. Of course, in these initiatives we are careful not to compromise reviewer confidentiality. Many reviewers see their work as a voluntary and often unseen part of their role as researchers. We are grateful to the time reviewers donate to our journals and the contribution they make.

If you are interested in becoming a reviewer for Laws, see the link at the bottom of the webpage http://www.mdpi.com/reviewers.

The following reviewed for Laws in 2016:

$\begin{array}{lll}\text { Abrams, Jamie R. } & \text { Clarke, Lynda } & \text { Gooding, Piers } \\ \text { Alaei, Kamiar } & \text { Clements, Luke } & \text { Guthrie, Robert } \\ \text { Atapattu, Sumudu } & \text { Clough, Beverley } & \text { Hamoudi, Haider Ala } \\ \text { Backhouse, Constance } & \text { Da Lomba, Sylvie } & \text { Harpur, Paul } \\ \text { Bahner, Julia } & \text { Dahlvik, Julia } & \text { Heinz, Andreas } \\ \text { Baines, Beverley } & \text { Dana, Genya } & \text { Henriksen, Tore } \\ \text { Barocas, Briana } & \text { De Beco, Gauthier } & \text { Hens, Kristien } \\ \text { Bartlett, Peter } & \text { Deem, Michael } & \text { Hernández, César } \\ \text { Baxi, Upendra } & \text { Depoorter, Ben } & \text { Cuauhtémoc García } \\ \text { Bernal, Paul } & \text { Downing, Steven } & \text { Hickey, Huhana } \\ \text { Blakeley, Georgina } & \text { Dudley, Michael } & \text { Higgins, Noelle } \\ \text { Blaser, Arthur } & \text { Easton, Catherine } & \text { Hollander-Blumoff, Rebecca } \\ \text { Boer, Ben } & \text { Ehrlich, J. Shoshanna } & \text { Hulme, Karen } \\ \text { Bonython, Wendy } & \text { England, Sarah } & \text { Jacquet, Jennifer } \\ \text { Boon-Kuo, Louise } & \text { Ferri, Delia } & \text { Jochnowitz, Leona D. } \\ \text { Brandt, Reuven } & \text { Fiore, Robin N. } & \text { Kaplan, Paul } \\ \text { Breger, Melissa L. } & \text { Fischer, Karla } & \text { Kelly, Fiona } \\ \text { Brophy, Lisa } & \text { Flynn, Eilionóir } & \text { Kett, Maria } \\ \text { Busby, Nicole } & \text { Foster, Stephen H. } & \text { Khan, Ummni } \\ \text { Byron, Christine } & \text { Gauderman, Kimberly } & \text { Kirakosyan, Lyusyena } \\ \text { Campbell, Marilyn } & \text { Gauthier, Ryan } & \text { Krajewska, Atina } \\ \text { Campie, Patricia E. } & \text { Gervais, Daniel J. } & \text { Krotoszynski, Ronald } \\ \text { Caranta, Roberto } & \text { Gibbons, Llewellyn Joseph } & \text { Kuiken, Todd } \\ \text { Carline, Anna } & \text { Glebbeek, Marie-Louise } & \text { Lamble, Sarah } \\ \text { Cashman, Peter } & \text { Goldblatt, Beth } & \text { Lankford, Adam } \\ \text { Chadwick, Ruth } & \text { Goldscheid, Julie } & \text { Lone, Fozia }\end{array}$


Lord, Janet E.

Luo, Pingping

Marouf, Fatma

Mason, Arthur

Meyers, Stephen

O'Connell, Karen

O'Donnell, Aisling

O'Mahony, Charles

Orlando, Emanuela

Paull, Megan

Peguera, Miquel

Perlin, Michael L.

Petersen, Carole J.

Petrikin, Joshua E.

Petrov, Andrey

Phillips, Scott

Pinto, Paula C.
Plomin, Robert

Prenzler, Tim

Quinn, Gerard

Richardson, Genevra

Rioux, Marcia H

Roberts, Jessica L.

Rooksby, Jacob H.

Ryan, Christopher J.

Seck, Sara

Seuba, Xavier

Shakespeare, Tom

Shanley, Mary L.

Skilton, Mark

Smith, Kevin

Snelling, Jeanne

Spencer, David

Stanley, Nicky
Steele, Linda

Steglich, Elissa

Stoett, Peter

Szmukler, George

Taeuber, Stacy

Thibault, Lucie

Thiele, Kathrin

Tignino, Mara

Vears, Danya

Vieten, Ulrike M.

Weber, Rolf H.

Weston, Maureen Arellano

Whyte, Kyle

Youm, Kyu Ho

(C) 2017 by the authors; licensee MDPI, Basel, Switzerland. This article is an open access article distributed under the terms and conditions of the Creative Commons Attribution (CC-BY) license (http://creativecommons.org/licenses/by/4.0/). 